\title{
Minimum degree conditions for small percolating sets in bootstrap percolation
}

\author{
Karen Gunderson* \\ Department of Mathematics \\ University of Manitoba \\ Winnipeg MB R3T 2N2, Canada \\ karen.gunderson@umanitoba.ca
}

Submitted: Mar 31, 2017; Accepted: May 8, 2020; Published: May 29, 2020

(C) The author. Released under the CC BY-ND license (International 4.0).

\begin{abstract}
The $r$-neighbour bootstrap process is an update rule for the states of vertices in which 'uninfected' vertices with at least $r$ 'infected' neighbours become infected and a set of initially infected vertices is said to percolate if eventually all vertices are infected. For every $r \geqslant 3$, a sharp condition is given for the minimum degree of a sufficiently large graph that guarantees the existence of a percolating set of size $r$. In the case $r=3$, for $n$ large enough, any graph on $n$ vertices with minimum degree $\lfloor n / 2\rfloor+1$ has a percolating set of size 3 and for $r \geqslant 4$ and $n$ large enough (in terms of $r$ ), every graph on $n$ vertices with minimum degree $\lfloor n / 2\rfloor+(r-3)$ has a percolating set of size $r$. A class of examples are given to show the sharpness of these results.
\end{abstract}

Mathematics Subject Classifications: 60K35, 05C35

\section{Introduction}

Bootstrap percolation is a model for the spread of an 'infection' in a network that was first introduced and investigated by Chalupa, Leath, and Reich [7] as a monotone model of the dynamics of ferromagnetism. Starting from a set of initially infected vertices in a graph, the infection spreads to new vertices depending on their number of infected neighbours. The focus in this paper will be on extremal problems related to these processes.

For any integer $r \geqslant 2$, the $r$-neighbour bootstrap process is an update rule for the states of vertices in a graph which are in one of two possible states at any given time: 'infected' or 'uninfected'. From an initial configuration of infected and uninfected vertices, the

\footnotetext{
*Supported by NSERC grant RGPIN-2016-05949.
} 
following state updates occur simultaneously and at discrete time steps: any uninfected vertex with at least $r$ infected neighbours becomes infected while infected vertices remain infected forever. To be precise, given a graph $G$ and a set $A \subseteq V(G)$ of 'initially infected' vertices, set $A_{0}=A$ and for every $t \geqslant 1$ define

$$
A_{t}=A_{t-1} \cup\left\{v \in V(G)|| N(v) \cap A_{t-1} \mid \geqslant r\right\} .
$$

The closure of $A$ is $\langle A\rangle_{r}=\cup_{t \geqslant 0} A_{t}$; the set of vertices that are eventually infected starting from $A=A_{0}$. The set $A_{t} \backslash A_{t-1}$ shall often be referred to as the vertices infected at time step $t$. The set $A$ is said to span $\langle A\rangle_{r}$. The set $A$ is called closed iff $\langle A\rangle_{r}=A$ and is said to percolate iff $\langle A\rangle_{r}=V(G)$.

While the focus of study for such processes is often the behaviour of initially infected sets that are chosen at random, a number of natural extremal problems arise. In this paper, the focus is on the size of a smallest percolating set. For other extremal problems, see $[3,5,6,15,17]$, for example. For any graph $G$ and $r \geqslant 2$, define the size of the smallest percolating set to be

$$
m(G, r)=\min \left\{|A| \mid A \subseteq V(G),\langle A\rangle_{r}=V(G)\right\} .
$$

One class of graphs that have received a great deal of attention in this area are the square grids. For any $n$ and $d$, let $[n]^{d}$ denote the $d$-dimensional $n \times n \times \cdots \times n$ grid. In the case that $r=2$, for all $n$ and $d$, the Balogh, Bollobás and Morris [2] showed that $m\left([n]^{d}, 2\right)=\lceil d(n-1) / 2\rceil+1$ (see also [1]). Pete (see [4]) gave a number of general results about the smallest percolating sets in grids with other thresholds and claimed that $m\left([n]^{d}, d\right)=n^{d-1}$ (a proof of which was given explicitly by Przykucki and Shelton [18]). In the case of hypercubes, $Q_{d}=[2]^{d}$, Morrison and Noel [16] confirmed a conjecture of Balogh and Bollobás [1], showing that for each fixed $r, m\left(Q_{d}, r\right)=\frac{(1+o(1))}{r}\left(\begin{array}{c}d \\ r-1\end{array}\right)$. A simpler proof of this was given by Hambardzumyan, Hatami, and Qian [13].

The size of minimum percolating sets in regular graphs have been examined by CojaOghlan, Feige, Krivelevich and Reichman [8] who gave bounds on $m(G, r)$ in a number of different cases in which $G$ is a regular graph satisfying various expansion properties. Bounds on the size of a minimum percolating set (or 'contagious set') in both binomial random graphs and random regular graphs have been given by Feige, Krivelevich, and Reichman [10] and Guggiola and Semerjian [12]. Minimum percolating sets in trees were investigated by Riedl [20].

In this note, we shall focus on the conditions for the minimum degree of a graph that imply the existence of a percolating set of the smallest possible size. It is clear that for any graph on at least $r$ vertices, $m(G, r) \geqslant r$. Throughout, $\delta(G)$ is used to denoted the minimum degree of a graph $G$.

Considering the degree sequence of a graph, Reichman [19] showed that for any any graph $G$ and threshold $r$, then

$$
m(G, r) \leqslant \sum_{v \in V(G)} \min \left\{1, \frac{r}{\operatorname{deg}(v)+1}\right\} .
$$


For any $d \geqslant r-1$, this upper bound is achieved by disjoint copies of cliques on $d+1$ vertices.

Freund, Poloczek, and Reichman [11] investigated Ore-type degree conditions for a graph that guarantee that $m(G, 2)=2$. Defining $\sigma_{2}(G)$ to be the minimum sum of degrees of non-adjacent vertices in $G$, they showed that for a graph on $n \geqslant 2$ vertices, if $\sigma_{2}(G) \geqslant n$, then $m(G, 2)=2$. Subsequently, Dairyko, Ferrara, Lidický, Martin, Pfender, and Uzzell [9] improved this result showing that, except for a list of exceptional graphs that they completely characterized, if $\sigma_{2}(G) \geqslant n-2$, then $m(G, 2)=2$. Their results show that the only graph with $\delta(G)=\lfloor|V(G)| / 2\rfloor$ and $m(G, 2)>2$ is the 5-cycle.

Freund, Poloczek, and Reichman [11] further showed that if $G$ is a graph on $n$ vertices with $\delta(G) \geqslant\left[\frac{(r-1)}{r} n\right]$, then $m(G, r)=r$. Though it is not stated in their paper, the proof idea in [11] can be used, with a small extra check, to show that for $n$ sufficiently large, and $\delta(G) \geqslant\lfloor n / 2\rfloor$, then $m(G, 2)=2$. This is best-possible as it is noted that for any $n$, a graph consisting of two disjoint cliques on $\lfloor n / 2\rfloor$ vertices and $\lceil n / 2\rceil$ vertices has minimum degree $\left\lfloor\frac{(2-1)}{2} n\right\rfloor-1$ and no set of size 2 that percolates in 2-neighbour bootstrap percolation.

In [11], the following examples are given to show that their conditions on the minimum degree are tight. For odd $r$, a clique on $n=r+1$ vertices with a perfect matching deleted has minimum degree $n-2=\left\lfloor\frac{r-1}{r} n\right\rfloor$ and no set of size $r$ percolates. These examples are restricted to a small number of vertices, depending on the infection parameter $r$. When $r \geqslant 3$ and the number of vertices is large relative to $r$, a different picture emerges and, in fact, when $n$ is large, any graph on $n$ vertices with a minimum degree that exceeds $n / 2$ by some constant that depends on $r$ will have a set of size $r$ that percolates in $r$-neighbour bootstrap percolation. The main result of this paper is the following.

Theorem 1. For any $r \geqslant 4$ and $n$ sufficiently large, if $G$ is a graph on $n$ vertices with $\delta(G) \geqslant\lfloor n / 2\rfloor+(r-3)$, then $m(G, r)=r$.

The result for the case $r=3$ is slightly different than the rest and is, perhaps, closer to the behaviour of the case $r=2$ examined in [11].

Theorem 2. For any $n \geqslant 30$, any graph $G$ on $n$ vertices with $\delta(G) \geqslant\lfloor n / 2\rfloor+1$ satisfies $m(G, 3)=3$.

Theorems 1 and 2, show that for any $r \geqslant 3$, there is a constant $c_{r}$ depending only on $r$ so that if $\delta(G) \geqslant\lfloor|V(G)| / 2\rfloor+c_{r}$, then $G$ has a percolating set of size $r$. When $|V(G)|$ is large, this is a substantially weaker condition than the results in [11] that required $\delta(G) \geqslant\lceil(r-1)|V(G)| / r\rceil$ for the same conclusion.

In the proofs to come for these theorems, no attempt has been made to optimize the possible lower bounds on $n$.

One obvious example of a graph with no set of size $r$ that percolates in $r$-neighbour bootstrap percolation is a graph consisting of two disjoint cliques of order $\lfloor n / 2\rfloor$ and $\lceil n / 2\rceil$ (as long as $n>r$ ). By adding some edges to such graphs, one can increase the minimum degree without creating any sets of size $r$ that percolate. In Section 2, examples 
are given of graphs on $n$ vertices with $\delta(G)=\lfloor n / 2\rfloor$ and $m(G, 3)>3$ and for every $r \geqslant 4$, examples of graphs with $\delta(G)=\lfloor n / 2\rfloor+(r-4)$ and $m(G, r)>r$. These examples show that Theorem 1 and Theorem 2 are sharp.

The different behaviour for the cases $r \leqslant 3$ and $r \geqslant 4$ is also characterized by these types of examples. Suppose that $G$ is a graph that consists of two cliques of equal size (on parts $A$ and $B$ ) with a $k$-regular bipartite graph between the two parts. Choose any 2 vertices $a_{1}, a_{2} \in A$. If $k \geqslant 2$, then there are vertices $b_{1}, b_{2} \in B$ with a path $a_{1}, b_{1}, a_{2}, b_{2}$ in the graph. If $a_{1}, a_{2}$ and any $r-2$ vertices in $B \backslash\left\{b_{1}, b_{2}\right\}$ are initially infected, then the infection spreads to $b_{1}, b_{2}$ and then all of $B$. If $k \geqslant r-2$, then all of $A$ is subsequently infected. The condition $k \geqslant \max \{2, r-2\}$ requires $r \geqslant 4$. Next, consider an initially infected set with only one vertex, $a$, in the set $A$. As long as $k \geqslant r-1, a$ together with any $r-1$ vertices in $B$ that are not adjacent to $a$ will percolate. If $r \leqslant 3$, then any percolating set of $r$ vertices will have at most 1 vertex in one of the parts $A$ or $B$. This forces a stronger condition on $k$ in these types of examples for the graphs to have a percolating set of size $r$. In Theorem 3, it is shown that unless the bipartite graph between $A$ and $B$ has short cycles, no improvement on $k$ is gained by having at least 3 infected vertices in each of $A$ and $B$. As will be seen in the proofs in Section 4, these examples are typical, though a larger class of graphs are considered for the proofs in which rather than having cliques on each part, the subgraphs are dense and instead of a regular bipartite graph between the two parts, on one side, vertices will have some small range of number of neighbours on the other side.

Throughout, the following notation is used. Given two disjoint sets of vertices $A$ and $B$ in a graph $G$, let $e(A, B)$ denote the number of edges with one endpoint in $A$ and the other in $B$. The subgraph of $G$ induced by the set $A$ is denoted by $G[A]$ and given two disjoint sets $A$ and $B$, let $G[A, B]$ denote the bipartite subgraph consisting of all the edges in $G$ with one endpoint in $A$ and the other in $B$. Given a set $A$ and a vertex $x$, let $\operatorname{deg}_{A}(x)$ be the number of neighbours of $x$ in the set $A$. The neighbourhood of a vertex $x$ in $G$ is denoted $N(x)$.

The remainder of the paper is organized as follows. In Section 2, we give the classes of graphs that show the sharpness of Theorem 1 and Theorem 2. In Section 3, it is shown that for all large graphs satisfying the degree conditions of Theorem 1 or Theorem 2 , every closed set is either relatively small, consists of around half the vertices, or is the set of all vertices. Using the existence of small complete bipartite subgraphs, it is shown that there is always a set of $r$ vertices whose closure is not too small. In Section 4, it is shown that graphs with closed sets consisting of nearly half the vertices are highly structured and that this structure can be exploited to find a percolating set of size $r$. Finally, in Section 5, some further open problems are given.

\section{Graphs with no small percolating sets}

The graphs described in this section showing the sharpness of Theorem 1 and Theorem 2 consist of two disjoint cliques, with a regular (or nearly-regular when the number of vertices is odd) bipartite graph between them that does not have small girth. Note that 
a bipartite graph $H$ with girth at least 6 as required by Theorem 3 is given with positive probability by taking a random $(r-3)$-regular bipartite graph on two vertex sets of size $n / 2$ as long as $n$ is sufficiently large (see, for example, results of Wormald [22] and [23]).

Theorem 3. For $r \geqslant 4$, let $n \geqslant 2(r-1)$ be even and suppose that $H$ is a $(r-3)$-regular bipartite graph on parts $A$ and $B$ of size $n / 2$ each such that $H$ has no 4-cycles. The graph $G$ consisting of $H$ together with a clique on the vertices of $A$ and a clique on the vertices of $B$ has $\delta(G)=n / 2+(r-4)$ and $m(G, r)>r$.

Proof. As the graph $G$ is $(n / 2+(r-4))$-regular, it remains only to show that no set of $r$ vertices percolates.

Let $X$ be any initially infected set of $r$ vertices in $G$ and set $|X \cap A|=k$. Note that since every vertex in $A$ has $r-3$ neighbours in $B$ and every vertex in $B$ has $r-3$ neighbours in $A$, if $k \leqslant 2$, then no vertex in $A \backslash X$ is ever infected, even if everything in $B$ is infected. Similarly, if $r-k \leqslant 2$, no vertex in $B \backslash X$ is ever infected.

We first use this observation to deal with some of the small values of $r$. For $r \in\{4,5\}$, if $k \geqslant 3$, then $r-k \leqslant r-3 \leqslant 2$. Thus, in the cases $r=4$ or $r=5$, it is immediate that $X$ does not percolate and so $m(G, r)>r$.

Next, consider the case that $r=6$. By the previous observation and relabelling $A$ and $B$ if necessary, assume that $3 \leqslant k \leqslant r-k \leqslant r-3$, so that we have $3=k=r-k$. If anything further is infected by $X$, say $a \in A$, then $a$ must be adjacent to all 3 elements of $X \cap B$. Since $H$ contains no copies of $C_{4}$, no other vertices in $A$ can be adjacent to all elements of $X \cap B$ and so there is at most one such $a \in A$.

If $a$ is the only vertex infected at time 1 , then no vertex in $B$ is adjacent to all elements of $X \cap A$ (or else it would have been infected in the first time step) and the only vertices adjacent to $a$ are those in $X \cap B$, which are already infected. Thus, nothing further is infected.

If two vertices are infected at the first time step, then since $H$ is $C_{4}$-free, it can only be that one $a \in A$ and one $b \in B$ are infected. That is, $a$ is adjacent to all elements in $X \cap B$ and $b$ is adjacent to all elements in $X \cap A$. At the second time step, any further vertex in $A$ is adjacent to 4 infected vertices in $A$, but not $b$ and at most one from $X \cap B$ (since otherwise, it would form a copy of $C_{4}$ with $a$ ). Thus, such a vertex does not become infected. Similarly, nothing in $B$ that is not already infected has more than 5 infected neighbours. Thus, $X$ does not percolate and so $m(G, 6)>6$.

Now we consider the most general case: $r \geqslant 7$. As above, let $X$ be any set of $r$ vertices in $G$, set $k=|X \cap A|$ and assume that $3 \leqslant k, r-k \leqslant r-3$.

First suppose that only vertices in one partition set, say $A$, are infected at the first time step. Since $H$ has no copy of $C_{4}$, there can be only one such vertex $x$ adjacent to all $r-k$ vertices in $X \cap B$. At the second time step, any uninfected vertex in $A$ has $k+1$ infected neighbours in $A$ and so can only be infected if it has $r-k-1 \geqslant 2$ neighbours in $X \cap B$. As this would create a $C_{4}$ with $x$, no vertex in $A$ can be infected in the second time step. Any vertex in $B$ that is infected at time 2 is in $N(x) \cap B \backslash X$. Set $N_{x}=N(x) \cap B \backslash X$ and note that $\left|N_{x}\right|=k-3$. If $k=3$, then no further vertices are infected and the process stops. If $k \geqslant 4$ and $y \in N_{x}$ is infected at the second time step, then $y$ has exactly $k-1$ 
(a)





Figure 1: Possible structures for the set $X$ when $r \geqslant 7$ if $(a)$ one vertex is infected in the first time step or $(b)$ two vertices are infected in the first time step. Shaded regions represent cliques.

neighbours in $X \cap A$ and there can only be one such vertex $y$ since a second would have two common neighbours with $y$ in $A(x$ and a vertex in $X \cap A)$. See Figure $1(a)$. At time step 3, any vertex in $A$ has at most $|(X \cap A) \cup\{x\}|=k+1$ infected neighbours in $A$ and at most 1 infected neighbour in $B$ (since two would create a $C_{4}$ with $x$ in $H$ ). Any vertex in $B$ has at most $|(X \cap B) \cup\{y\}|=(r-k)+1$ infected neighbours in $B$ and is either adjacent to $x$ and at most one vertex from $X \cap A \backslash N(y)$ or else at most two vertices from $X \cap A$. Thus, any uninfected vertex is adjacent to at most $\max \{k+2, r-k+3\}$ infected vertices. Since $k \geqslant 4$, then $\max \{k+2, r-k+3\} \leqslant r-1$ and so no further vertices are infected. As long as $n \geqslant 2(r-1)$, then there are still uninfected vertices and so the set $X$ does not percolate.

Next, suppose that $x \in A$ and $y \in B$ are both infected at the first time step. Without loss of generality, assume that $3 \leqslant k \leqslant r-k \leqslant r-3$. As before, there can be only one vertex in each partition set that is infected in the first time step. Set $N_{x}=B \cap N(x) \backslash X$ and $N_{y}=A \cap N(y) \backslash X$ so that $\left|N_{x}\right|=k-3$ and $\left|N_{y}\right|=r-k-3$. See Figure $1(b)$. At time step 2, any vertex in $N_{y}$ is adjacent to $|(X \cap A) \cup\{x\}|=k+1$ infected vertices in $A$ at most 2 vertices in $B$ ( $y$ and at most one from $X \cap B$ ). Since $k+1+2=k+3 \leqslant r-1$, then such a vertex in $N_{y}$ is not infected. If $k=3$, then there are no vertices in $N_{x}$ and so any vertex in $B$ has at most $r-k+2$ infected neighbours $(r-k+1$ neighbours in $(X \cap B) \cup\{x\}$ and at most one in $X \cap A$ as two would create a $C_{4}$ with $\left.y\right)$. Since $r-k+1 \leqslant r-1$, no further vertex in $B$ is infected. If $k \geqslant 4$, then any vertex in $N_{x}$ is adjacent to at most $(r-k)+1$ infected neighbours in $B$ and at most 2 in $A$ since it can have at most one neighbour in $X \cap A$. For $k \leqslant r / 2$ and $r \geqslant 7$, we have $r-k+3 \leqslant r-1$ and so such a vertex in $N_{y}$ is not infected.

In all cases, the bootstrap process halts with not all vertices infected since $n \geqslant 2(r-1)$. As the set $X$ was arbitrary, then $m(G, r)>r$.

The construction in Theorem 3 can be extended to an odd number of vertices as follows.

Corollary 4. For every $r \geqslant 4$ and $n$ sufficiently large, there is a graph $G$ on $n$ vertices with $\delta(G)=\lfloor n / 2\rfloor+(r-4)$ with $m(G, r)>r$. 
Proof. If $n$ is even, let $G$ be given by Theorem 3. If $n$ is odd, let $G_{1}$ be the graph on $n+1$ vertices given by Theorem 3 and define a graph $G$ by deleting one vertex from $G_{1}$. The vertices of $G$ are partitioned into a set, $A$, of size $\lceil n / 2\rceil$ that form a clique and a set, $B$, of size $\lfloor n / 2\rfloor$ that each form a clique. Vertices in $A$ have degree at least $\lceil n / 2\rceil-1+(r-3)-1=\lfloor n / 2\rfloor+(r-4)$ while vertices in $B$ have degree exactly $\lfloor n / 2\rfloor-1+(r-3)=\lfloor n / 2\rfloor+(r-4)$. If $G$ had a percolating set of size $r$ for $r$-neighbour bootstrap percolation, then this same set would percolate in $G_{1}$ since the additional vertex is joined to at least $r$ neighbours in $B$. As this would contradict the fact that $m\left(G_{1}, r\right)>r$, then $m(G, r)>r$ also.

The case $r=3$ has a different behaviour than larger values of $r$. The proof that the example has no small percolating sets is closely related to the corresponding proofs for $r \in\{4,5\}$.

Theorem 5. For any even $n \geqslant 4$, let $A=[1, n / 2]$ and $B=[n / 2+1, n]$ and let $G$ be the graph given by a complete graph on $A$, a complete graph on $B$ and a perfect matching between $A$ and $B$. Then, $\delta(G)=n / 2$ and $m(G, 3)>3$.

Proof. Let $X$ be any set of 3 vertices in $G$. Note that either $|X \cap A| \leqslant 1$ or else $|X \cap B| \leqslant 1$. Suppose, without loss of generality that $|X \cap A| \leqslant 1$. Even if every vertex in $B$ becomes infected, any uninfected vertex in $A$ has at most 2 infected neighbours: any vertex in $X \cap A$ and the single neighbour in $B$. Thus, these vertices never become infected and so $X$ does not percolate.

The same argument as that given in the proof of Corollary 4 extends Theorem 5 to all $n \geqslant 4$.

Corollary 6. For any $n \geqslant 4$, there exists a graph $G$ with $\delta(G)=\lfloor n / 2\rfloor$ and $m(G, 3)>3$.

Note that the graph described in Theorem 5 was also used [11] where it was called $D C_{n}$ and it was noted, in relation to 2-neighbour bootstrap percolation, that this graph has sets of size 2 whose closure is of size $n / 2$, while there are other sets of size two that percolate.

This concludes the descriptions of constructions and in the subsequent sections, it is shown that large graphs with minimum degree one larger (for a fixed $n$ and $r$ ) than those in Theorems 3 and 5 do have small percolating sets. No attempt has been made here to classify all extremal examples.

\section{Sets with large closure}

Before proceeding to the proofs of the main theorems, we give a number of results about the size of the closures of sets in $r$-neighbour bootstrap percolation. In particular, the goal is to show that the closures of any set in graphs satisfying a minimum degree condition can only have a small number of different sizes.

The following straightforward lemma uses a minimum degree condition to show that any large set will percolate. This will be used repeatedly in arguments to come. 
Lemma 7. For any $r \geqslant 3, k \geqslant 1$, let $G$ be a graph on $n$ vertices with $\delta(G) \geqslant\lfloor n / 2\rfloor+k$. Every set $A \subseteq V(G)$ with $|A| \geqslant\left\lceil\frac{n}{2}\right\rceil+(r-k-1)$ satisfies $\langle A\rangle_{r}=V(G)$.

Proof. For every $x \in A^{c}$, since $x$ has at most $\left|A^{c}\right|-1=n-|A|-1$ neighbours within $A^{c}$, then

$$
\begin{aligned}
\operatorname{deg}_{A}(x) & =\operatorname{deg}(x)-\operatorname{deg}_{A^{c}}(x) \\
& \geqslant\left\lfloor\frac{n}{2}\right\rfloor+k-n+|A|+1 \\
& \geqslant\left\lfloor\frac{n}{2}\right\rfloor+k-n+\left(\left\lceil\frac{n}{2}\right\rfloor+r-k-1\right)+1 \\
& =r .
\end{aligned}
$$

Thus, as every vertex in $A^{c}$ has at least $r$ neighbours in $A$, if the set $A$ is initially infected, the remainder of the graph becomes infected in one time step.

There are two different cases for the choice of $k$ in Lemma 7 used here. In the case $r=3$ with $k=1$, this lemma states that if $\delta(G) \geqslant\lfloor n / 2\rfloor+1$, then any set of size $\lceil n / 2\rceil+1$ percolates. For all $r \geqslant 4$, taking $k=r-3$, the lemma shows that for $\delta(G)=\lfloor n / 2\rfloor+r-3$, any set of size $\lceil n / 2\rceil+2$ percolates.

In the following proposition, we consider large graphs with a given minimum degree condition. Edge-counting is used to show that any set that is closed is either relatively small or else contains nearly half of the vertices of the graph. This includes the possibility that the set percolates.

Proposition 8. Let $r \geqslant 3$, set $k=\max \{1, r-3\}$ and let $G$ be a graph on $n$ vertices with $n \geqslant 10 r$ and $\delta(G) \geqslant\lfloor n / 2\rfloor+k$. If $A \subseteq V(G)$ is such that $\langle A\rangle_{r}=A$, then either $|A| \leqslant 2(r-1)$ or else $|A| \geqslant\lfloor n / 2\rfloor-\min \{1, r-3\}$.

Proof. Let $A$ be a set of vertices with $\langle A\rangle_{r}=A$ and set $|A|=\ell$. The proof proceeds by counting the edges with one endpoint in $A$ and the other in $A^{c}$ in two different ways.

Since any vertex in $A$ has at most $\ell-1$ neighbours within the set $A$, any $x \in A$ has at least $\delta(G)-\ell+1$ neighbours in the set $A^{c}$. Thus,

$$
e\left(A, A^{c}\right)=\sum_{x \in A} \operatorname{deg}_{A^{c}}(x) \geqslant \ell(\delta(G)-\ell+1) \geqslant \ell(\lfloor n / 2\rfloor-\ell+k+1) .
$$

On the other hand, since $\langle A\rangle_{r}=A$, every vertex in $A^{c}$ can have at most $r-1$ neighbours in the set $A$. Thus,

$$
e\left(A, A^{c}\right)=\sum_{x \in A^{c}} \operatorname{deg}_{A}(x) \leqslant(r-1)\left|A^{c}\right|=(r-1)(n-\ell) .
$$

Combining the inequalities (1) and (2) and rearranging gives that

$$
0 \leqslant \ell^{2}-\ell\left(\left\lfloor\frac{n}{2}\right\rfloor+k+r\right)+(r-1) n .
$$


To conclude the proof, the righthand side of inequality (3) is analyzed to determine those values of $\ell$ for which it can be positive and so deduce bounds on $|A|$. Towards this end, define $D(\ell)=\ell^{2}-\ell(\lfloor n / 2\rfloor+k+r)+(r-1) n$, which is the righthand side of inequality (3). Substituting $\ell=2 r-1$ into $D(\ell)$ gives

$$
\begin{aligned}
& D(2 r-1)=(2 r-1)^{2}-(2 r-1)\lfloor n / 2\rfloor-(2 r-1)(k+r)+n(r-1) \\
& =(2 r-1)(r-k-1)+(r-1)\left(n-2\left\lfloor\frac{n}{2}\right\rfloor\right)-\left\lfloor\frac{n}{2}\right\rfloor \\
& \leqslant(2 r-1)(r-k-1)+(r-1)-\left\lfloor\frac{n}{2}\right\rfloor \\
& = \begin{cases}7-\left\lfloor\frac{n}{2}\right\rfloor & \text { if } r=3 \\
5 r-3-\left\lfloor\frac{n}{2}\right\rfloor & \text { if } r \geqslant 4\end{cases} \\
& <0
\end{aligned}
$$

since for $n \geqslant 10 r-4$, then $\lfloor n / 2\rfloor>5 r-3$. Furthermore, substituting $\ell=2 r-2$ gives, for all $n$,

$$
\begin{aligned}
D(2 r-2) & =(2 r-2)^{2}-2(r-1)\lfloor n / 2\rfloor-(2 r-2)(k+r)+n(r-1) \\
& =(2 r-2)(r-k-2)+(r-1)\left(n-2\left\lfloor\frac{n}{2}\right\rfloor\right) \geqslant 0 .
\end{aligned}
$$

Similarly, substituting $\ell=\lfloor n / 2\rfloor-2$ gives

$$
\begin{aligned}
& D(\lfloor n / 2\rfloor-2)=\lfloor n / 2\rfloor^{2}-4\lfloor n / 2\rfloor+4-\lfloor n / 2\rfloor^{2}-(k+r-2)\lfloor n / 2\rfloor \\
&+2(k+r)+n(r-1) \\
&=(r-1)(n-2\lfloor n / 2\rfloor)-(k-r+4)\lfloor n / 2\rfloor+2(k+r+2) \\
& \leqslant 2 k+3 r+3-\lfloor n / 2\rfloor<0
\end{aligned}
$$

for $n \geqslant 10 r$. Next consider the result of substituting $\ell=\lfloor n / 2\rfloor-1$,

$$
\begin{aligned}
D(\lfloor n / 2\rfloor-1)= & \lfloor n / 2\rfloor^{2}-2\lfloor n / 2\rfloor+1-\lfloor n / 2\rfloor^{2}-(k+r-1)\lfloor n / 2\rfloor \\
& +(k+r)+n(r-1) \\
= & n(r-1)-(k+r+1)\lfloor n / 2\rfloor+(k+r+1) \\
= & \begin{cases}2 n-5\lfloor n / 2\rfloor+5 & \text { if } r=3 \\
(r-1)(n-2\lfloor n / 2\rfloor)+2 r-2 & \text { if } r \geqslant 4 .\end{cases}
\end{aligned}
$$

Thus, when $r=3$, and $n \geqslant 16, D(\lfloor n / 2\rfloor-1)<0$ whereas for $r \geqslant 4$ and all $n$, we have $D(\lfloor n / 2\rfloor-1) \geqslant 0$. Finally, consider $D(\lfloor n / 2\rfloor)$ in the case that $r=3$ :

$$
\begin{aligned}
D(\lfloor n / 2\rfloor) & =\lfloor n / 2\rfloor^{2}-\lfloor n / 2\rfloor^{2}-4\lfloor n / 2\rfloor+2 n \\
& =2(n-2\lfloor n / 2\rfloor) \geqslant 0 .
\end{aligned}
$$

Note that $D$ is a quadratic function in $\ell$ with a unique minimum and satisfying $D(2 r-$ $2) \geqslant 0, D(2 r-1)<0$. When $r=3$, since $D(\lfloor n / 2\rfloor-1)<0$ and $D(\lfloor n / 2\rfloor) \geqslant 0$, then 
if $D(\ell) \geqslant 0$, then either $\ell \leqslant 4$ or else $\ell \geqslant\lfloor n / 2\rfloor$. When $r \geqslant 4$, since $D(\lfloor n / 2\rfloor-2)<0$ and $D(\lfloor n / 2\rfloor-1) \geqslant 0$, then if $D(\ell) \geqslant 0$, either $\ell \leqslant 2(r-1)$ or else $\ell \geqslant\lfloor n / 2\rfloor-1$. This completes the proof.

In summary, Lemma 7 and Proposition 8 together show that if $G$ is a graph on $n$ vertices with $\delta(G) \geqslant\lfloor n / 2\rfloor+\max \{1, r-3\}$, then any set of $r$ vertices either percolates, spans a set of size at most $2(r-1)$ or else spans a set of cardinality close to $n / 2$. In the case that $r=3$, any large closed set that does not percolate has cardinality $\lfloor n / 2\rfloor$ or $\lceil n / 2\rceil$. In the case that $r \geqslant 4$, if $A$ is a large closed set that does not percolate, then $\lfloor n / 2\rfloor-1 \leqslant|A| \leqslant\lceil n / 2\rceil+1$.

In order to address the existence of small closed sets of vertices in the graph, note that for $r$ fixed and $n$ large enough, the Kövari-Sós-Turán theorem [14] implies that a graph on $n$ vertices with minimum degree $\delta(G) \geqslant\lfloor n / 2\rfloor+(r-3)$ contains complete bipartite subgraphs of the form $K_{r, r-1}$ which give a subgraph on $2 r-1$ vertices with $m\left(K_{r, r-1}, r\right)=r$. For the sake of completeness, the following pair of lemmas with standard proofs make this precise.

Lemma 9. For $n \geqslant 6$, if $G$ is a graph on $n$ vertices with $\delta(G) \geqslant\lfloor n / 2\rfloor+1$, then any vertex of $G$ is contained in a copy of $K_{2,3}$.

Proof. Let $x$ be any vertex in $G$. If $x$ is adjacent to all other vertices, then for any $y \neq x$, the common neighbourhood of $x$ and $y$ has at least $\lfloor n / 2\rfloor \geqslant 3$ vertices and these together with $x$ and $y$ form a copy of $K_{2,3}$. Otherwise, let $z$ be any non-neighbour of $x$. Then the common neighbourhood of $x$ and $z$ has at least $2(\lfloor n / 2\rfloor+1)-(n-2)=2\lfloor n / 2\rfloor-n+4 \geqslant 3$ vertices and these together with $x$ and $z$ form a copy of $K_{2,3}$.

Lemma 10. For each $r \geqslant 3$ and $n \geqslant(r-1) 2^{r-1}+4$, if $G$ is a graph on $n$ vertices with $\delta(G) \geqslant\lfloor n / 2\rfloor+(r-3)$, then $G$ contains a copy of $K_{r, r-1}$.

Proof. The proof proceeds by counting copies of stars of the form $K_{1, r-1}$. Define the set

$$
S=\{(x, A)|x \in V(G),| A \mid=r-1, A \subseteq N(x)\} .
$$

Then, counting elements of $S$ by the first coordinate, as long as $\lfloor n / 2\rfloor+(r-2) \geqslant r-1$, then

$$
\begin{aligned}
|S| & =\sum_{x \in V}\left(\begin{array}{c}
\operatorname{deg}(x) \\
r-1
\end{array}\right) \\
& \geqslant \sum_{x \in V}\left(\begin{array}{c}
\lfloor n / 2\rfloor+(r-3) \\
r-1
\end{array}\right) \\
& =n\left(\begin{array}{c}
\lfloor n / 2\rfloor+(r-3) \\
r-1
\end{array}\right) \\
& \geqslant \frac{n}{(r-1) !}\left(\frac{n-1}{2}+(r-3)\right) \cdots\left(\frac{n-1}{2}+1\right)\left(\frac{n-1}{2}\right)\left(\frac{n-1}{2}-1\right)
\end{aligned}
$$




$$
\begin{aligned}
& \geqslant \frac{n}{(r-1) !} \cdot \frac{(n-1)(n-2) \cdots(n-r+2)}{2^{r-1}} \cdot(n-3) \\
& \geqslant \frac{n-3}{2^{r-1}}\left(\begin{array}{c}
n \\
r-1
\end{array}\right)>(r-1)\left(\begin{array}{c}
n \\
r-1
\end{array}\right) .
\end{aligned}
$$

As there are $\left(\begin{array}{c}n \\ r-1\end{array}\right)$ possible choices for the second coordinate of elements of $S$, by the pigeonhole principle, there is a set $A \subseteq V(G)$ of $r-1$ vertices with at least $r$ common neighbours. These $r$ vertices, together with $A$ contain a copy of $K_{r, r-1}$ in the graph.

Thus, when $n$ is sufficiently large, any graph on $n$ vertices with minimum degree $\lfloor n / 2\rfloor+(r-3)$ has a set of size $r$ whose span contains at least $2 r-1$ vertices and hence by Proposition 8, contains at least $\lfloor n / 2\rfloor-1$ vertices. If the span has more than $\lceil n / 2\rceil+1$ vertices, then by Lemma 11, the set percolates.

What remains to show is that if such a graph contains a set $A$ with around half the vertices in $G$ and $\langle A\rangle_{r}=A$, then $G$ contains some set of size $r$ that percolates.

\section{Structure of large closed sets}

In this section, we show that if a graph on $n$ vertices has minimum degree $\lfloor n / 2\rfloor+$ $\max \{r-3,1\}$ and $A$ is a set with close to half the vertices of $G$ and $\langle A\rangle_{r}=A$, then enough structural information about $G$ can be deduced to show that there is some set of size $r$ that percolates, completing the proof of Theorem 1. As the minimum degree conditions for the case $r=3$ are different from all others, these are dealt with separately.

Before proceeding with these results, a few straightforward lemmas are recorded to be used repeatedly. The first shows that if the minimum degree of a graph is large enough, not only is there a set of $r$ vertices that percolates in $r$-neighbour bootstrap percolation, but, in fact, any set of $r$ vertices will percolate.

Lemma 11. Let $k \geqslant 0, r \geqslant 3$ and $n \geqslant k(r+1)-1$. For any graph $G$ on $n$ vertices with $\delta(G) \geqslant n-k$ and any set $A \subseteq V(G)$ of $r$ vertices, $\langle A\rangle_{r}=V(G)$.

Proof. As each vertex in $G$ has at most $k-1$ non-neighbours, the vertices in the set $A$ have at least $n-|A|-(k-1)|A|=n-k r$ common neighbours. Since $n-k r \geqslant k-1$, when the set $A$ is initially infected at least $k-1$ further vertices are infected at the first time step. At this point, any uninfected vertex is adjacent to at least $(r+k-1)-(k-1)=r$ infected vertices and so becomes infected in the second time step. Thus, the set $A$ percolates.

The next lemma gives bounds on the possible distribution of degrees between various parts of a graph when there is a closed set with around half of the vertices. Two very similar cases are handled separately in the statement of the lemma because of different estimates used in the different cases in later proofs.

Lemma 12. Let $r \geqslant 3$ and let $G$ be a graph on $n$ vertices with $\delta(G) \geqslant\lfloor n / 2\rfloor+\max \{1, r-$ 3\}. Let $A \subseteq V(G)$ be such that $\langle A\rangle_{r}=A$ and let $i \in\{0,1\}$. 
Case 1: If $|A|=\lfloor n / 2\rfloor-i$, then for $x \in A$, $\operatorname{deg}_{A^{c}}(x) \geqslant \max \{2, r-2\}+i$ and for $y \in A^{c}$,

$$
\begin{aligned}
\operatorname{deg}_{A}(y) & \geqslant r-3-i, \text { and } \\
\operatorname{deg}_{A^{c}}(y) & \geqslant\left(\left|A^{c}\right|-1\right)-(\min \{2, r-2\}+i) .
\end{aligned}
$$

Case 2: If $|A|=\lceil n / 2\rceil+i$, then for $x \in A$

$$
\operatorname{deg}_{A^{c}}(x) \geqslant \begin{cases}\max \{2, r-2\}-i & \text { if } n \text { is even } \\ \max \{1, r-3\}-i & \text { if } n \text { is odd }\end{cases}
$$

and for any $y \in A^{c}$,

$$
\begin{aligned}
\operatorname{deg}_{A}(y) & \geqslant \max \{2, r-2\}+i, \text { and } \\
\operatorname{deg}_{A^{c}}(y) & \geqslant\left(\left|A^{c}\right|-1\right)-(\min \{1, r-3\}-i)
\end{aligned}
$$

Proof. Case 1: Suppose that $A$ is closed with $|A|=\lfloor n / 2\rfloor-i$. Then, $\left|A^{c}\right|=\lceil n / 2\rceil-i$. For any $x \in A$,

$$
\begin{aligned}
\operatorname{deg}_{A^{c}}(x) & \geqslant \delta(G)-(|A|-1) \geqslant\lfloor n / 2\rfloor+\max \{1, r-3\}-\lfloor n / 2\rfloor+i+1 \\
& =\max \{2, r-2\}+i
\end{aligned}
$$

Similarly, for $y \in A^{c}$,

$$
\begin{aligned}
\operatorname{deg}_{A^{c}}(y) & \geqslant \delta(G)-\left(\left|A^{c}\right|-1\right) \geqslant\lfloor n / 2\rfloor+\max \{1, r-3\}-\lceil n / 2\rceil-i+1 \\
& =\lfloor n / 2\rfloor-\lceil n / 2\rceil+\max \{1, r-3\}+1-i \\
& \geqslant-1+r-3+1-i=r-3-i
\end{aligned}
$$

Since $A$ is closed, then $\operatorname{deg}_{A}(y) \leqslant r-1$ and so

$$
\begin{aligned}
\operatorname{deg}_{A}(y) & \geqslant \delta(G)-(r-1) \geqslant\lfloor n / 2\rfloor+\max \{1, r-3\}-r+1 \\
& =\left|A^{c}\right|-1-\lceil n / 2\rceil-i+1+\lfloor n / 2\rfloor+\max \{1, r-3\}-r+1 \\
& \geqslant\left|A^{c}\right|-1-(\max \{1, r-3\}-r+1-i) \\
& =\left(\left|A^{c}\right|-1\right)-(\min \{2, r-2\}+i) .
\end{aligned}
$$

This completes the proof in the first case.

Case 2: Suppose that $A$ is closed with $|A|=\lceil n / 2\rceil+i$. The proof in this case is similar to that of Case 1 . For any $x \in A$,

$$
\begin{aligned}
\operatorname{deg}_{A^{c}}(x) & \geqslant \delta(G)-(|A|-1) \geqslant \max \{1, r-3\}+1-(\lceil n / 2\rceil-\lfloor n / 2\rfloor)-i \\
& = \begin{cases}\max \{2, r-2\}-i & \text { if } n \text { is even, } \\
\max \{1, r-3\}-i & \text { if } n \text { is odd. }\end{cases}
\end{aligned}
$$

For any $y \in A^{c}, \operatorname{deg}_{A}(y) \geqslant \delta(G)-\left(\left|A^{c}\right|-1\right) \geqslant \max \{2, r-2\}+i$. Since $A$ is closed, $\operatorname{deg}_{A}(y) \leqslant r-1$ and so $\operatorname{deg}_{A^{c}}(y) \geqslant \delta(G)-(r-1) \geqslant\left(\left|A^{c}\right|-1\right)-(\min \{1, r-3\}-i)$. 
The following structural fact about graphs with a large closed set is used repeatedly in the proofs of Proposition 18 and Proposition 19. The proof follows immediately from Lemma 12 in the case $|A|=\lceil n / 2\rceil+1$.

Fact 13. For any $r \geqslant 4, n \geqslant 2 r$ and $G$ a graph on $n$ vertices with $\delta(G) \geqslant\lfloor n / 2\rfloor+r-3$, let $A$ be a set with $|A|=\lceil n / 2\rceil+1$ and $\langle A\rangle_{r}=A$. Then $G\left[A^{c}\right]$ is a complete graph and every vertex has exactly $r-1$ neighbours in $A$.

\subsection{Threshold $r=3$}

In this subsection, it is shown that if any set of size 3 in a graph with minimum degree $\lfloor n / 2\rfloor+1$ spans either $\lfloor n / 2\rfloor$ or $\lceil n / 2\rceil$ vertices, then some set of 3 vertices percolates in 3 -neighbour bootstrap percolation. The two different cases that arise when $n$ is odd are handled in separate propositions.

Proposition 14. Let $G$ be a graph on $n \geqslant 13$ vertices with $\delta(G) \geqslant\lfloor n / 2\rfloor+1$ and let $A \subseteq V(G)$ be such that $|A|=\lfloor n / 2\rfloor$. If $\langle A\rangle_{3}=A$, then $m(G, 3)=3$.

Proof. By Lemma 12 in Case 1 with $|A|=\lfloor n / 2\rfloor$, for any $x \in A, \operatorname{deg}_{A^{c}}(x) \geqslant 2$. Furthermore, for any $y \in A^{c}, \operatorname{deg}_{A^{c}}(y) \geqslant\left(\left|A^{c}\right|-1\right)-1$. Then, by Lemma 11 applied to $G\left[A^{c}\right]$, since $n \geqslant 6$, any set of 3 vertices in $A^{c}$ infects all of $A^{c}$.

Set $A_{3}=\left\{x \in A \mid \operatorname{deg}_{A^{c}}(x) \geqslant 3\right\}$. If $A_{3} \neq \emptyset$, then for any three vertices $a, b, c \in A^{c}$,

$$
\left|\langle\{a, b, c\}\rangle_{3}\right| \geqslant\left|A^{c}\right|+\left|A_{3}\right| \geqslant\lceil n / 2\rceil+1
$$

Then, by Lemma $7,\langle\{a, b, c\}\rangle_{3}=V(G)$ and this is a percolating set of size 3 .

Thus, assume that $A_{3}=\emptyset$ and hence $G[A]$ is a complete graph with every vertex having exactly 2 neighbours in $A^{c}$. By Lemma 12 , every $y \in A^{c} \operatorname{has} \operatorname{deg}_{A}(y) \geqslant 1$. Set $B_{1}=\left\{y \in A^{c} \mid \operatorname{deg}_{A}(y)=1\right\}$. Since $A$ is closed, every vertex in $A^{c}$ has at most 2 neighbours in $A$. Then,

$$
2\lfloor n / 2\rfloor=e\left(A, A^{c}\right)=\left|B_{1}\right|+2\left(\lceil n / 2\rceil-\left|B_{1}\right|\right)=2\lceil n / 2\rceil-\left|B_{1}\right|
$$

which implies that $\left|B_{1}\right|=2(\lceil n / 2\rceil-\lfloor n / 2\rfloor) \leqslant 2$.

Pick any $y \in A^{c} \backslash B_{1}$ and let $a, b$ be its 2 neighbours in $A$. Let $z \in A^{c}$ be any other neighbour of $a$ and choose any $c \in A \backslash\{a, b\}$. Consider the effect of initially infecting the set $\{c, y, z\}$; see Figure 2. Then, $a$ is adjacent to all 3 and becomes infected in the first time step. Then, $b$ is adjacent to $a, c$, and $y$ and so becomes infected by the second time step. Since $G[A]$ is complete and contains three infected vertices, all remaining vertices of $A$ are infected by the third time step. Finally, any vertex in $A^{c} \backslash B_{1}$ is adjacent to at least one of $y$ and $z$ and has two further infected neighbours in $A$ and so becomes infected by time step 4. Finally, if there is a vertex in $B_{1}$, it is adjacent to all elements of $A^{c}$ and has one infected neighbour in $A$ and so also becomes infected by step 4 . Therefore, $\{c, y, z\}$ is a percolating set of size 3 , completing the proof.

Proposition 15. Let $n \geqslant 13$ be odd and let $G$ be a graph on $n$ vertices with $\delta(G) \geqslant \frac{n+1}{2}$ and let $A \subseteq V(G)$ be such that $|A|=\frac{n+1}{2}$. If $\langle A\rangle_{3}=A$, then $m(G, 3)=3$. 


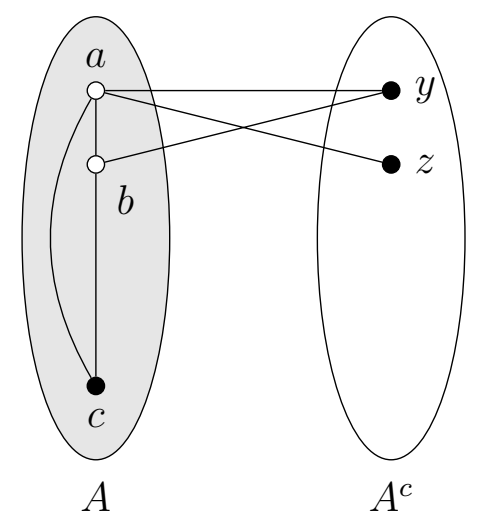

Figure 2: Case for $r=3$ and $A_{3}=\emptyset$ in the proof of Proposition 14.

Proof. Applying Lemma 12 with Case 2 shows that every $y \in A^{c}$ has at least 2 neighbours in $A$ and at least $\frac{n+1}{2}-2=\left|A^{c}\right|-1$ neighbours in $A^{c}$. That is, $G\left[A^{c}\right]$ is a complete graph and every vertex has exactly 2 neighbours in $A$.

Also, by Lemma 12, any vertex in $A$ has at least 1 neighbour in $A^{c}$. Set $A_{3}=$ $\left\{x \in A \mid \operatorname{deg}_{A^{c}}(x) \geqslant 3\right\}$. If $\left|A_{3}\right| \geqslant 2$, then any three vertices in $A^{c}$ span at least $\left|A^{c}\right|+\left|A_{3}\right| \geqslant \frac{n-1}{2}+2=\lceil n / 2\rceil+1$ vertices and so percolate by Lemma 7 . If $A_{3}=\emptyset$, then $\left\langle A^{c}\right\rangle_{3}=A^{c}$ and so by Proposition 14, $G$ has a percolating set of size 3 .

Assume now that $\left|A_{3}\right|=1$. Note that every vertex in $A \backslash A_{3}$ has either 1 or 2 neighbours in $A^{c}$ and at most one non-neighbour in $A$. Thus, by Lemma 11, any set of size 3 in $A \backslash A_{3}$ eventually infects all of $A \backslash A_{3}$. If $\left\langle A \backslash A_{3}\right\rangle_{3}=A \backslash A_{3}$, then again by Proposition 14, $G$ has a percolating set of size 3. If $\left\langle A \backslash A_{3}\right\rangle \supsetneq A$, then by Lemma 7 , everything is eventually infected and so $G$ has a percolating set of size 3 .

Therefore, assume that $\left|A_{3}\right|=1$ and that $\left\langle A \backslash A_{3}\right\rangle_{3}=A$. Let $x$ be any vertex in $A \backslash A_{3}$ and let $a$ be one its neighbours in $A^{c}$. Let $y, z \in A \backslash A_{3}$ be any two neighbours of $x$ and consider the effect of initially infecting $\{a, y, z\}$. Then since $x$ is adjacent to all 3 , it is infected in the first time step. By assumption, $\langle\{x, y, z\}\rangle_{3}=A$ and so $\langle\{a, y, z\}\rangle_{3} \supseteq$ $A \cup\{a\}$, which is a set of size $\lceil n / 2\rceil+1$. Thus, by Lemma 7 , the set $\{a, y, z\}$ percolates.

In all cases, the graph $G$ contains 3 vertices that percolate and so $m(G, 3)=3$.

With these two results, the proof of Theorem 2 now follows.

Proof of Theorem 2. Let $n \geqslant 30$ and let $G$ be a graph on $n$ vertices with $\delta(G) \geqslant\lfloor n / 2\rfloor+1$. By Lemma 9, $G$ contains a copy of $K_{2,3}$. Let $A$ be a set of 3 vertices in one of the partition classes in any copy of $K_{2,3}$, since $\left|\langle A\rangle_{r}\right| \geqslant 5>2(3-1)$, by Lemma 7 and Proposition 8, either $A$ percolates or else $\left|\langle A\rangle_{3}\right| \in\{\lfloor n / 2\rfloor,\lceil n / 2\rceil\}$. By Propositions 14 and 15, if $\left|\langle A\rangle_{3}\right| \in\{\lfloor n / 2\rfloor,\lceil n / 2\rceil\}$, then $G$ contains some set of size 3 that percolates. Thus, $m(G, 3)=3$, which completes the proof. 


\subsection{Threshold $r \geqslant 4$}

In this subsection, we consider bootstrap processes with infection threshold $r \geqslant 4$ and give the proof of Theorem 1. The proof uses more steps than that for the corresponding result for $r=3$ because of the weaker result for Proposition 8 in the case $r \geqslant 4$. Throughout the proofs, the aim will be to either find a set of size $r$ that spans at least $\lceil n / 2\rceil+2$ vertices (and hence percolates by Lemma 7 ) or else to deduce enough structural information about the graph to reduce the problem to a few special cases.

Proposition 16. Let $r \geqslant 4$, let $n$ be sufficiently large, and let $G$ be a graph on $n$ vertices with $\delta(G) \geqslant\lfloor n / 2\rfloor+(r-3)$. If there is a set $A \subseteq V(G)$ with $|A|=\lfloor n / 2\rfloor-1$ and $\langle A\rangle_{r}=A$, then $m(G, r)=r$.

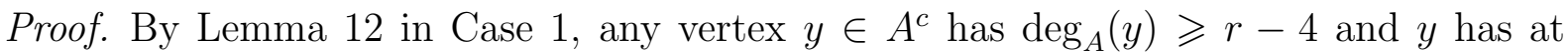
most 3 non-neighbours in the set $A^{c}$. Thus, by Lemma 11, any set of $r$ vertices in $A^{c}$ infects all of $A^{c}$. Furthermore, by Lemma 12, any vertex in $A$ has at least $r-1 \geqslant 1$ neighbours in $A^{c}$ and so $e\left(A, A^{c}\right) \neq 0$. Let $b \in A^{c}$ be any vertex with a neighbour $a \in A$. Let $v_{1}, v_{2}, \ldots, v_{r-1}$ be any neighbours of $b$ in $A^{c}$ and consider initially infecting the set $\left\{a, v_{1}, v_{2}, \ldots, v_{r-1}\right\}$. Since $b$ is adjacent to all $r$ infected vertices, it is infected at the first time step. Then, by the previous comment, $\left\{b, v_{1}, \ldots, v_{r-1}\right\}$ internally spans the entire set $A^{c}$. Since,

$$
\left|\left\langle\left\{a, v_{1}, v_{2}, \ldots, v_{r-1}\right\}\right\rangle_{r}\right| \geqslant\left|A^{c} \cup\{a\}\right|=\lceil n / 2\rceil+1+1=\lceil n / 2\rceil+2,
$$

then by Lemma 7 , the set $\left\{a, v_{1}, v_{2}, \ldots, v_{r-1}\right\}$ percolates and so $m(G, r)=r$.

Proposition 17. Let $r \geqslant 4$, let $n$ be sufficiently large, and let $G$ be a graph on $n$ vertices with $\delta(G) \geqslant\lfloor n / 2\rfloor+(r-3)$. If there is a set $A \subseteq V(G)$ with $|A|=\lfloor n / 2\rfloor$ and $\langle A\rangle_{r}=A$, then $m(G, r)=r$.

Proof. By Lemma 12 in Case 1 , for any $y \in A^{c}, \operatorname{deg}_{A}(y) \geqslant r-3$ and $y$ has at most 2 non-neighbours within $A^{c}$. Thus, by Lemma 11, any $r$ vertices in $A^{c}$ infect all of $A^{c}$.

If the graph $G\left[A, A^{c}\right]$ contains a copy of $K_{2,2}$ with vertices $a, b \in A$ and $x, y \in A^{c}$, let $v_{1}, v_{2}, \ldots, v_{r-2}$ be any $r-2$ common neighbours of $x$ and $y$ in $A^{c}$ and consider initially infecting the set $\left\{a, b, v_{1}, v_{2}, \ldots, v_{r-2}\right\}$, as in Figure $3(a)$. The vertices $x$ and $y$ are infected in the first time step and subsequently all vertices in $A^{c}$ are infected. Since at least $\left|A^{c}\right|+2=\lceil n / 2\rceil+2$ vertices are infected, the set percolates by Lemma 7 .

Now, assume that the graph $G\left[A, A^{c}\right]$ contains no copy of $K_{2,2}$. By Lemma 12 , every vertex in $A$ has at least $r-2$ neighbours in $A^{c}$, and so $e\left(A, A^{c}\right) \geqslant(r-2)\lfloor n / 2\rfloor$. Since every vertex in $A^{c}$ has at most $(r-1)$ neighbours in $A$, there are at most $\frac{1}{2}(\lceil n / 2\rceil+r-2)$ vertices $y \in A^{c}$ with $\operatorname{deg}_{A}(y)=r-3$. Since this is less than the total number of vertices in $A^{c}$, let $x \in A^{c}$ be a vertex with $\operatorname{deg}_{A}(x)=i \in\{r-2, r-1\}$ and let $a_{1}, a_{2}, \ldots, a_{i}$ be its neighbours in $A$. Note that $i \geqslant 2$. As each $a_{j}$ has at least $r-2 \geqslant 2$ neighbours in $A^{c}$, for each $j \leqslant i$, let $b_{j} \in A^{c} \backslash\{x\}$ be a neighbour of $a_{j}$. Since $G\left[A, A^{c}\right]$ contains no copy of $K_{2,2}$ all of the vertices $\left\{b_{1}, b_{2}, \ldots, b_{i}\right\}$ are distinct. Since the vertex $x$ has at most $\lceil n / 2\rceil-1-(\lfloor n / 2\rfloor+r-3-i) \leqslant i-(r-3)$ non-neighbours in $A^{c}$ and $i-(r-3) \leqslant i-1$, 


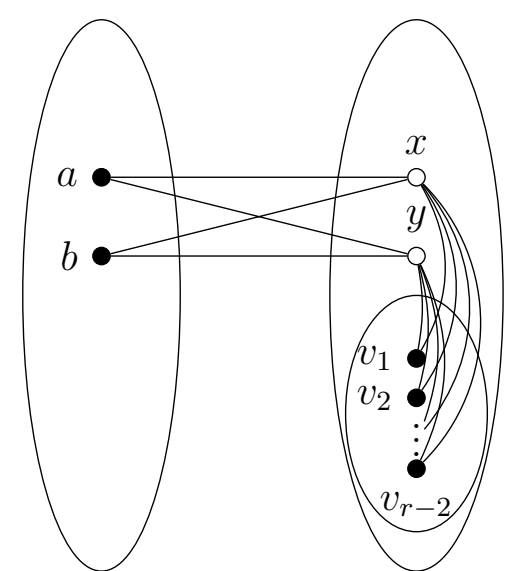

(a)
A
$A^{c}$

(b)

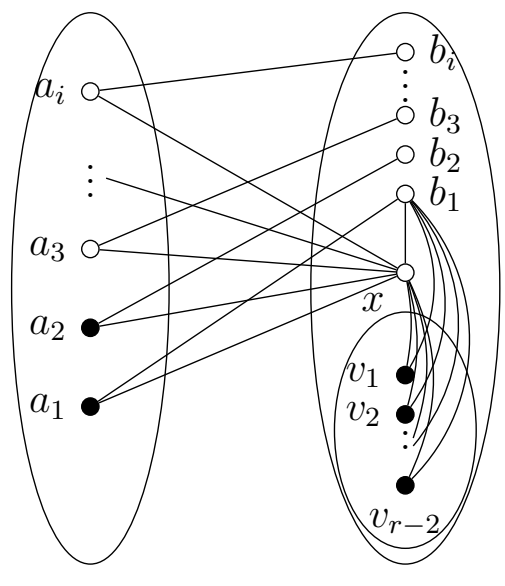

A

Figure 3: (a) $G\left[A, A^{c}\right]$ contains a copy of $K_{2,2} ;(b) G\left[A, A^{c}\right]$ is $K_{2,2}$-free

then $x$ is adjacent to at least one vertex in $\left\{b_{1}, b_{2}, \ldots, b_{i}\right\}$. Without loss of generality, suppose that $x$ is adjacent to $b_{1}$. Let $v_{1}, v_{2}, \ldots, v_{r-2}$ be any common neighbours of $x$ and $b_{1}$ in $A^{c}$ and consider initially infecting the set $\left\{a_{1}, a_{2}, v_{1}, v_{2}, \ldots, v_{r-2}\right\}$, as in Figure $3(b)$. The vertex $x$ is infected in the first time step, and $b_{1}$ by the second time step. Then, $A^{c}$ is internally spanned by $\left\{x, b_{1}, v_{1}, \ldots, v_{r-2}\right\}$ and since

$$
\left|\left\langle\left\{a_{1}, a_{2}, v_{1}, v_{2}, \ldots, v_{r-2}\right\}\right\rangle_{r}\right| \geqslant\left|A^{c} \cup\left\{a_{1}, a_{2}\right\}\right|=\lceil n / 2\rceil+2,
$$

then by Lemma 7, all vertices are eventually infected and so $m(G, r)=r$.

The aim in all of the proofs of this section is to use the structural information about the graphs to find a set of $r$ vertices that internally spans at least $\lceil n / 2\rceil+2$ vertices (and hence percolates). In some circumstances, finding many sets whose span is $\lceil n / 2\rceil+1$ can be quite useful as Fact 13 provides a great deal of information about structure regarding such sets.

Proposition 18. Let $r \geqslant 4$, let $n$ be sufficiently large and odd, and let $G$ be a graph on $n$ vertices with $\delta(G) \geqslant \frac{n-1}{2}+(r-3)$. If there is a set $A \subseteq V(G)$ with $|A|=\left\lceil\frac{n}{2}\right\rceil=\frac{n+1}{2}$ and $\langle A\rangle_{r}=A$, then $m(G, r)=r$.

Proof. By Lemma 12 in Case 2, any $y \in A^{c} \operatorname{has} \operatorname{deg}_{A}(y) \geqslant r-2$ and at most 1 nonneighbour in $A^{c}$. As before, by Lemma 11, any set of $r$ vertices in $A^{c}$ infects all of $A^{c}$, at least.

Furthermore, every vertex in $A$ has at least $r-3 \geqslant 1$ neighbours in $A^{c}$. Set $A_{r}=$ $\left\{x \in A \mid \operatorname{deg}_{A^{c}}(x) \geqslant r\right\}$. Note that since $r\left|A_{r}\right| \leqslant e\left(A, A^{c}\right) \leqslant(r-1)\left|A^{c}\right|$, then $\left|A_{r}\right| \leqslant$ $\frac{(r-1)}{r} \cdot \frac{(n-1)}{2} \leqslant \frac{n+1}{2}-(2 r+1)$ and so $\left|A \backslash A_{r}\right| \geqslant 2 r+1$. Any vertex in $A \backslash A_{r}$ has at most 2 non-neighbours in $A$ and so any set of size $r$ in $A \backslash A_{r}$ infects the remainder of $A \backslash A_{r}$ by Lemma 11. 


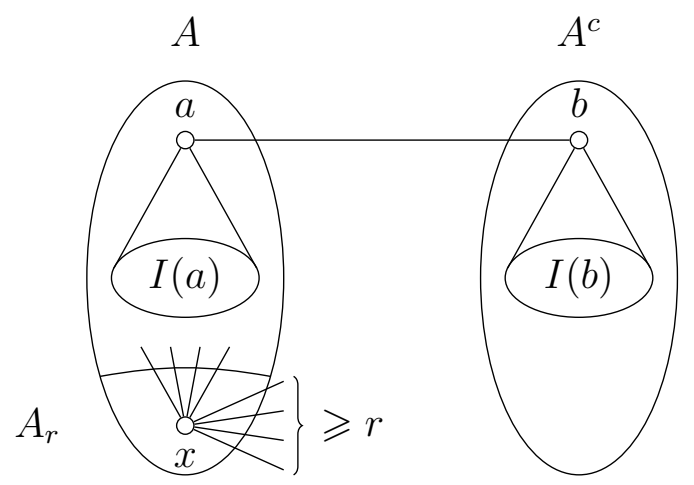

Figure 4: The sets $I(a)$ and $I(b)$ for an edge $\{a, b\}$.

If $A_{r}=\emptyset$, then $\left\langle A^{c}\right\rangle_{r}=A^{c}$, but since $\left|A^{c}\right|=\frac{n-1}{2}=\lfloor n / 2\rfloor$, then by Proposition 17, there is a set of size $r$ that percolates.

If $\left|A_{r}\right| \geqslant 2$, then choose any element $a \in A \backslash A_{r}$, let $b \in A^{c}$ be any neighbour of $a$ and let $v_{1}, v_{2}, \ldots, v_{r-1}$ be any $r-1$ neighbours of $b$ in $A^{c}$. Then, letting $B=\left\{a, v_{1}, v_{2}, \ldots, v_{r-1}\right\}$ be the set of initially infected vertices, $B$ infects $b$ and so with $r$ infected vertices in $A^{c}$, $\left|\langle B\rangle_{r}\right| \geqslant\left|A^{c} \cup A_{r} \cup\{a\}\right| \geqslant \frac{n-1}{2}+2+1=\lceil n / 2\rceil+2$ and so by Lemma $7, B$ percolates.

Suppose now that $\left|A_{r}\right|=1$ and let $A_{r}=\{x\}$. If $x$ has fewer than $r$ neighbours in $A$, then $A \backslash\{x\}$ is a closed set of size $\frac{n-1}{2}$ and so by Proposition 17, $G$ contains a set of size $r$ that percolates. Therefore, assume that $x$ has at least $r$ neighbours in $A$. The remainder of the proof involves considering many different sets of size $r$ and showing that if none percolate, then Fact 13 can be used to deduce sufficient structural information about $G$ to find a small percolating set.

For every vertex $a \in V(G) \backslash\{x\}$, choose a set, denoted $I(a)$ of $r-1$ neighbours of $a$ so that, if $a \in A \backslash\{x\}$, then $I(a) \subseteq A \backslash\{x\}$ and if $a \in A^{c}$, then $I(a) \subseteq A^{c}$. Every vertex in $A$ has at least one neighbour in $A^{c}$ and since $\left|A_{r}\right|=1$, then every vertex in $A^{c}$ has $r-2 \geqslant 2$ neighbours in $A$, at least one of which is in $A \backslash A_{r}$. For any pair $\{a, b\} \in E(G)$ with $a \in A \backslash A_{r}$ and $b \in A^{c}$, then

$$
\begin{aligned}
& \langle\{b\} \cup I(a)\rangle_{r} \supseteq A \cup\{b\}, \text { and } \\
& \langle\{a\} \cup I(b)\rangle_{r} \supseteq A^{c} \cup A_{r} \cup\{a\} .
\end{aligned}
$$

See Figure 4. Since each of the sets $\{b\} \cup I(a)$ and $\{a\} \cup I(b)$ each span a set of size at least $\frac{n+1}{2}+1=\frac{n-1}{2}+2$, either one of them percolates, or else by Fact 13, for every $a \in A \backslash A_{r}$, the graph induced by $G$ on $A \backslash\{a, x\}$ is a clique with every vertex having exactly $r-1$ neighbours in $A^{c} \cup\{a, x\}$ and similarly, for every $b \in A^{c}$, the set $A^{c} \backslash\{b\}$ induces a clique with every vertex having $r-1$ neighbours in $A \cup\{b\}$.

Note that any graph on at least 3 vertices with the property that deleting any vertex gives a clique is itself a clique. Thus, each of $G[A \backslash\{x\}]$ and $G\left[A^{c}\right]$ is a complete graph where every vertex in $A \backslash\{x\}$ has exactly $r-2$ neighbours in $A^{c} \cup\{x\}$ and every vertex in $A^{c}$ has exactly $r-2$ neighbours in $A$. 


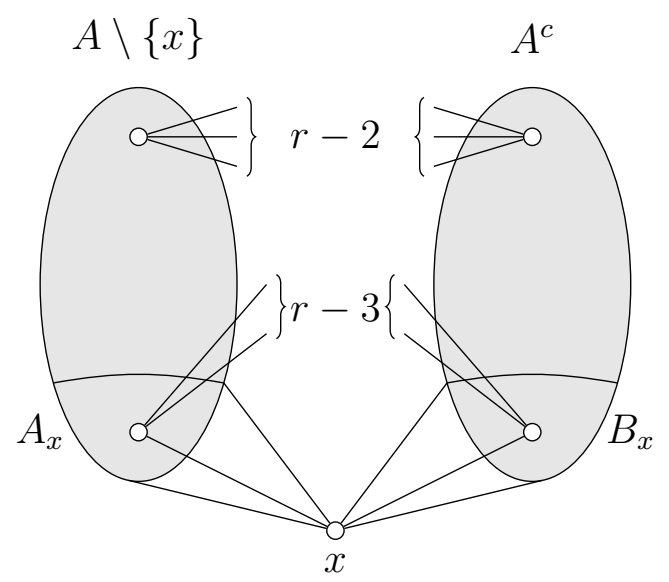

Figure 5: Structure of the graph if no set $\{a\} \cup I(b)$ or $\{b\} \cup I(a)$ percolates.

Set $A_{x}=A \cap N(x), A_{1}=A \backslash(\{x\} \cup N(x)), B_{x}=A^{c} \cap N(x)$ and $B_{1}=A^{c} \backslash N(x)$. By assumption, $\left|A_{x}\right|,\left|B_{x}\right| \geqslant r$. Note that every vertex in $A_{1}$ has $r-2 \geqslant 2$ neighbours in $A^{c}$ and every vertex in $A_{x}$ has $r-3 \geqslant 0$ neighbours in $A^{c}$; see Figure 5.

If any vertex $a \in A \backslash\{x\}$ has two neighbours $b_{1}, b_{2} \in B_{x}$, then let $\left\{v_{1}, v_{2}, \ldots, v_{r-2}\right\}$ be any $r-2$ vertices in $A_{x} \backslash\{a\}$ and consider initially infecting $\left\{b_{1}, b_{2}, v_{1}, v_{2}, \ldots, v_{r-2}\right\}$. Both $x$ and $a$ are adjacent to all infected vertices and so become infected at the first time step. Thereafter, the remainder of $A_{x}$ is infected and hence all of $A$. Since at least $\left|A \cup\left\{b_{1}, b_{2}\right\}\right|=\lceil n / 2\rceil+2$ vertices are infected, the set percolates, by Lemma 7 .

If any vertex $a \in A \backslash\{x\}$ has two neighbours $b_{1}, b_{2} \in A^{c}$ with $b_{1} \in B_{1}$, then since $b_{1}$ has at least $r-2 \geqslant 2$ neighbours in $A \backslash\{x\}$, let $c \in A \backslash\{a, x\}$ be any other neighbour of $b_{1}$. Let $v_{1}, v_{2}, \ldots, v_{r-2} \in A^{c} \backslash\left\{b_{1}, b_{2}\right\}$ be any $r-2$ vertices in $A^{c}$ and consider initially infecting the set $\left\{a, c, v_{1}, v_{2}, \ldots, v_{r-2}\right\}$. In the first step $b_{1}$ is infected, then $b_{2}$ and subsequently the remainder of $A^{c}$ and so also $x$. As at least $\left|A^{c} \cup\{a, c, x\}\right|=\lceil n / 2\rceil+2$ vertices are infected, the set percolates, by Lemma 7 .

By symmetry, the same is true for any vertex in $A^{c}$ with two neighbours in $A_{x}$ or else two neighbours in $A \backslash\{x\}$, one of which is in $A_{1}$.

For any $r \geqslant 5$, every vertex in $A_{1} \cup A_{x}$ has at least $r-3 \geqslant 2$ neighbours in $A^{c}$ and so either some vertex has 2 neighbours in $B_{x}$ or 2 neighbours one of which is in $B_{1}$. In either case, there is some set of size $r$ that percolates.

The only remaining case is when $r=4$ and there are no vertices in $A \backslash\{x\}$ with two neighbours in $A^{c}$ and similarly, no vertices in $A^{c}$ with two neighbours in $A \backslash\{x\}$. That is, $A_{1}=B_{1}=\emptyset$ and $G$ consists of a clique on $A_{x}$, a clique on $B_{x}$, all vertices in $A_{x} \cup B_{x}$ joined to $x$ and a perfect matching between $A_{x}$ and $B_{x}$, as in Figure 6 . Since $(n-1) / 2 \geqslant 4$, choose $a, b \in A_{x}$ and $c, d \in B_{x}$ with $c, d \notin N(a) \cup N(b)$ and initially infect the set $\{a, b, c, d\}$. The vertex $x$ is infected at the first time step. At the second time step, the neighbours of $a$ and $b$ in $B_{x}$ and the neighbours of $c$ and $d$ in $A_{x}$ are infected and then all remaining vertices are infected in the third time step.

This completes the proof in the case that $\langle A\rangle_{r}=A$ and $|A|=\left\lceil\frac{n}{2}\right\rceil$. 


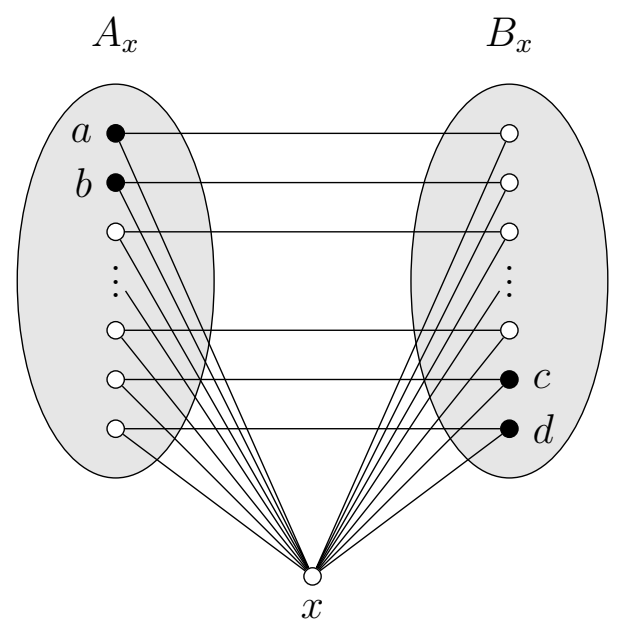

Figure 6: Case where $r=4$ and every vertex in $A \backslash\{x\}$ has only one neighbour in $A^{c}$.

The final remaining case to be dealt with is the following.

Proposition 19. Let $r \geqslant 4, n$ be sufficiently large, and let $G$ be a graph on $n$ vertices with $\delta(G) \geqslant\lfloor n / 2\rfloor+(r-3)$. If there is a set $A \subseteq V(G)$ with $|A|=\lceil n / 2\rceil+1$ and $\langle A\rangle_{r}=A$, then $m(G, r)=r$.

Proof. By Fact 13, the set $A^{c}$ induces a complete graph with every vertex having exactly $r-1$ neighbours in $A$. By Lemma 12 , any vertex $x \in A$ has

$$
\operatorname{deg}_{A^{c}}(x) \geqslant\left\{\begin{array}{ll}
r-3 & \text { if } n \text { is even, } \\
r-4 & \text { if } n \text { is odd }
\end{array} .\right.
$$

As in previous proofs, set $A_{r}=\left\{x \in A \mid \operatorname{deg}_{A^{c}}(x) \geqslant r\right\}$. Again, if $A_{r}=\emptyset$, then $A^{c}$ is a closed set of size $\lfloor n / 2\rfloor-1$ and so by Proposition 16, there is a percolating set of size $r$. If $\left|A_{r}\right| \geqslant 4$, then any set of size $r$ in $A^{c}$ spans $A^{c} \cup A_{r}$ which has $\lfloor n / 2\rfloor-1+4 \geqslant\lceil n / 2\rceil+2$ vertices and hence percolates by Lemma 7 .

Thus, assume that $A_{r} \neq \emptyset$ and $\left|A_{r}\right| \leqslant 3$. Every vertex in $A \backslash A_{r}$ has at most 3 nonneighbours in $A$. If $A_{r} \nsubseteq\left\langle A \backslash A_{r}\right\rangle_{r}$, then there is a closed set that is smaller than $A$ and so by one of Propositions 16, 17, or 18, $G$ has a percolating set of size $r$. Therefore, assume that $\left\langle A \backslash A_{r}\right\rangle_{r}=A$.

Note that since

$$
(r-1)(\lfloor n / 2\rfloor-1)=e\left(A, A^{c}\right) \geqslant r\left|A_{r}\right|
$$

then $\left|A_{r}\right| \leqslant \frac{(r-1)}{r}(\lfloor n / 2\rfloor-1) \leqslant\lceil n / 2\rceil-(r+3)$ as long as $n \geqslant 2\left(r^{2}+2 r+1\right)$.

If there is any vertex $a \in A \backslash A_{r}$ with a neighbour $b \in A^{c}$, then since $a$ has at most 3 non-neighbours in $A$, there are at least $r-1$ neighbours of $a$ in $A \backslash A_{r}$. Let $v_{1}, v_{2}, \ldots, v_{r-1}$ be any neighbours of $a$ in $A \backslash A_{r}$. Since the set $\left\{b, v_{1}, v_{2}, \ldots, v_{r-1}\right\}$ infects $a$ and hence all of $A \backslash A_{r}$ and subsequently $A_{r}$, the closure of this set has at least $|A|+1=\lceil n / 2\rceil+2$ vertices and hence the set percolates by Lemma 7 . 
Since $\left|A_{r}\right| \leqslant 3$, the only case in which there can be no edges between $A \backslash A_{r}$ and $A^{c}$ is when $r=4, n$ is odd, every vertex in $A^{c}$ has $r-1=3$ neighbours in $A_{r}$ and $G\left[A \backslash A_{r}\right]$ is a complete graph with all vertices in $A \backslash A_{r}$ adjacent to every vertex in $A_{r}$. In this case, $\left|A_{r}\right|=3$ and the graph is as in Figure 7. Then any set consisting of two vertices from $A \backslash A_{r}$ and two vertices from $A^{c}$ will infect all of $A_{r}$ and subsequently the remainder of the graph.

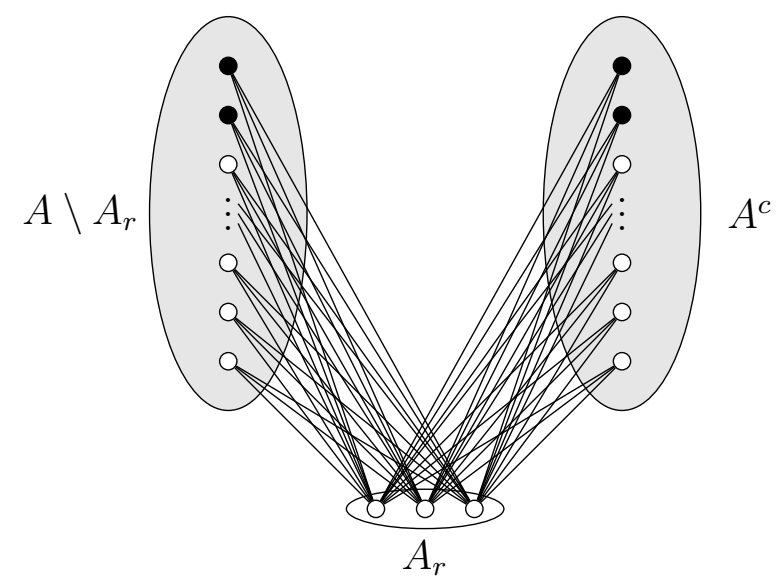

Figure 7: $r=4$ and no edges between $A \backslash A_{r}$ and $A^{c}$.

In all cases, there is some set of $r$ vertices that percolates and so $m(G, r)=r$.

The proof of Theorem 1 can now be completed.

Proof of Theorem 1. Let $n$ be large enough to apply the lemmas and propositions given previously and let $G$ be a graph on $n$ vertices with $\delta(G) \geqslant\lfloor n / 2\rfloor+(r-3)$. By Lemma 10, $G$ contains a copy of $K_{r, r-1}$ and the $r$ vertices in one partition set, $A$, have closure $\left|\langle A\rangle_{r}\right| \geqslant 2 r-1>2(r-1)$. By Lemma 7 and Proposition 8, either $A$ percolates or else $\left|\langle A\rangle_{r}\right| \in[\lfloor n / 2\rfloor-1,\lceil n / 2\rceil+1]$. If $\left|\langle A\rangle_{r}\right| \in[\lfloor n / 2\rfloor-1,\lceil n / 2\rceil+1]$, then by Proposition 16, 17,18 , or $19, G$ contains a percolating set of size $r$.

\section{Open problems}

There are a number of natural questions related to the results in this paper that remain open. One could ask for the conditions on $\delta(G)$ that guarantee $m(G, r) \leqslant k$ for a fixed $k \geqslant r+1$. Following the line of inquiry in [9] and [11], one might consider the lower bounds on $\sigma_{2}(G)$ that guarantee that $m(G, r)=r$ for $r \geqslant 3$. After this paper was submitted, answers to these questions for $k \leqslant 2 r-2$ were given by Wesolek [21].

A problem that may be quite technical would be the characterization of those small graphs for which $\delta(G)=\lfloor n / 2\rfloor+\min \{1, r-3\}$ but $m(G, r)>r$. 


\section{Acknowledgements}

I would like to thank the referee for their careful reading of the paper and many helpful suggestions that improved the presentation.

\section{References}

[1] J. Balogh, B. Bollobás, Bootstrap percolation on the hypercube, Probab. Theory Related Fields 134 (2006), 624-648.

[2] J. Balogh, B. Bollobás, R. Morris, Bootstrap percolation in high dimensions, Combin. Probab. Comput. 19 (2010), 643-692.

[3] J. Balogh, B. Bollobás, R. Morris, O. Riordan, Linear algebra and bootstrap percolation, J. Combin. Theory Ser. A 119 (2012), 1328-1335.

[4] J. Balogh, G. Pete, Random disease on the square grid, Random Structures Algorithms 13 (1998), 409-422.

[5] F. S. Benevides, M. Przykucki, On slowly percolating sets of minimal size in bootstrap percolation, Electron. J. Combin. 20 (2013), \#P46.

[6] F. Benevides, M. Przykucki, Maximum percolation time in two-dimensional bootstrap percolation, SIAM J. Discrete Math. 29 (2015), no. 1, 224-251.

[7] J. Chalupa, P.L. Leath, G.R. Reich, Bootstrap percolation on a Bethe lattice, Journal of Physics C 12 (1979), L31-L35.

[8] A. Coja-Oghlan, U. Feige, M. Krivelevich, D. Reichman, Contagious sets in expanders, Proceedings of the Twenty-Sixth Annual ACM-SIAM Symposium on Discrete Algorithms (2015), 1953-1987.

[9] M. Dairyko, M. Ferrara, B. Lidický, R. R. Martin, F. Pfender, A. J. Uzzell, Ore and Chvátal-type degree conditions for bootstrap percolation from small sets, J. Graph Theory 94 (2019), 252-266.

[10] U. Feige, M. Krivelevich, D. Reichman, Contagious sets in random graphs, Ann. Appl. Probab. 27 (2017), 2675-2697.

[11] D. Freund, M. Poloczek, D. Reichman, Contagious sets in dense graphs, European J. Combin. 68 (2018), 66-78.

[12] A. Guggiola, G. Semerjian, Minimal contagious sets in random regular graphs, J. Stat. Phys. 158 (2015), 300-358.

[13] L. Hambardzumyan, H. Hatami, Y. Qian, Polynomial method and graph bootstrap percolation, J. Combin. Theory Ser. A 174 (2020), 105253.

[14] T. Kövari, V. T. Sós, P. Turán, On a problem of K. Zarankiewicz, Colloquium Math. 3 (1954), 50-57.

[15] R. Morris, Minimal percolating sets in bootstrap percolation, Electron. J. Combin. 16 (2009), \#R2. 
[16] N. Morrison, J. A. Noel, Extremal bounds for bootstrap percolation in the hypercube, J. Combin. Theory Ser. A 156 (2018), 61-84.

[17] M. Przykucki, Maximal percolation time in hypercubes under 2-bootstrap percolation, Electron. J. Combin., 19(2) (2012), \#P41.

[18] M. Przykucki, T. Shelton, Smallest percolating sets in bootstrap percolation on grids, arXiv preprint (2019), arXiv: 1907.01940 [math.CO].

[19] D. Reichman, New bounds for contagious sets, Discrete Math. 312 (2012), 1812-1814.

[20] E. Riedl, Largest and smallest minimal percolating sets in trees, Electron. J. Combin. 19(1) (2012), \#P64.

[21] A. Wesolek, Bootstrap percolation in Ore-type graphs, arXiv preprint (2019), arXiv: 1909. 04649 [math.CO].

[22] N. C. Wormald, Some problems in the enumeration of labelled graphs, PhD thesis, University of Newcastle, 1978.

[23] N. C. Wormald, Models of random regular graphs, Surveys in combinatorics, London Math. Soc. Lecture Note Ser., 267, Cambridge Univ. Press, Cambridge, 1999, pp. 239-298. 